OPEN ACCESS

Edited by:

Lisandro M. Sakata,

Federal University of Paraná, Brazil

Reviewed by:

Monisha Nongpiur:

Singapore Eye Research Institute

(SERI), Singapore

Jayter Paula,

University of São Paulo, Brazil

*Correspondence:

Shengjie Li

lishengjie6363020@163.com

Wenjun Cao

wgkjyk@aliyun.com

Specialty section

This article was submitted to

Ophthalmology,

a section of the journal

Frontiers in Medicine

Received: 19 October 2020

Accepted: 04 January 2021

Published: 02 February 2021

Citation:

Shao M, Li Y, Teng J, Li S and Cao W (2021) Association Between Serum Lipid Levels and Patients With Primary Angle-Closure Glaucoma in China: A Cross Sectional, Case-Control Study. Front. Med. 8:618970. doi: 10.3389/fmed.2021.618970

\section{Association Between Serum Lipid Levels and Patients With Primary Angle-Closure Glaucoma in China: A Cross Sectional, Case-Control Study}

\author{
Mingxi Shao, Yingzhu Li, Jisen Teng, Shengjie $\mathrm{Li}^{\star}$ and Wenjun Cao* \\ Clinical Laboratory, Eye \& Ear Nose Throat (ENT) Hospital, Fudan University, Shanghai, China
}

Objective: To evaluate the serum lipid levels of patients with primary angle-closure glaucoma (PACG) and to investigate the relationship between serum lipid levels and PACG.

Methods: In this cross-sectional, case-control study, a total of 320 PACG subjects and 242 age- and sex-matched control subjects were recruited.Serum high-density lipoprotein cholesterol (HDL-C), low-density lipoprotein cholesterol (LDL-C), small dense LDL-C (SDLDL-C), triglyceride (TRIG), and cholesterol (CHOL) levels were measured using enzymatic colorimetry. Serum apolipoprotein A (APOA), apolipoprotein $B(A P O B)$, apolipoprotein $E(A P O E)$, and lipoprotein(a) $(\mathrm{LPa})$ levels were measured by immunoturbidimetry.

Results: The serum LDL-C, TRIG, HDL-C, APOE, LPa, CHOL, APOB, and APOA levels were significantly higher $(p<0.05)$ in the PACG group than in the control group. Multiple liner regression analyses revealed that there was a statistically correlation between HDL-C levels and mean deviation MD $(B=0.389, P=0.002,95 \%$ confidence interval $[\mathrm{Cl}]=-1.249$ to -0.624$)$; LDL-C levels and $\mathrm{MD}(B=0.190$, $P=0.019,95 \% \mathrm{Cl}=-5.632$ to -1.306$)$; and $\mathrm{CHOL}$ levels and $\mathrm{MD}(B=0.364$, $P=0.27,95 \% \mathrm{Cl}=-7.727$ to -1.839$)$. Logistic regression analyses showed that high serum HDL-C (odds ratio $[\mathrm{OR}]=11.01,95 \% \mathrm{Cl}=5.616-21.587)$, LDL-C (OR = 1.330, $95 \% \mathrm{Cl}=1.079-1.640), \quad S D L D L-C(O R=1.007,95 \% \mathrm{Cl}=1.005-1.008), \quad$ APOA $(\mathrm{OR}=13.621,95 \% \mathrm{Cl}=7.251-25.591), \mathrm{APOB}(\mathrm{OR}=2.243,95 \% \mathrm{Cl}=1.060-4.732)$, $\mathrm{LPa}(\mathrm{OR}=0.999, \quad 95 \% \mathrm{Cl}=0.998-1.00)$, and $\mathrm{CHOL} \quad(\mathrm{OR}=1.131,95 \%$ $\mathrm{Cl}=1.005-1.326$ ) levels were significantly associated with PACG.

Conclusions: High serum HDL-C, LDL-C, APOA, APOB, LPa, and CHOL levels were associated with PACG.

Keywords: primary angle-closure glaucoma, serum lipid concentration, high-density lipoprotein cholesterol, triglyceride, low-density lipoprotein cholesterol, cholesterol 


\section{INTRODUCTION}

Glaucoma is a multifactor-induced progressive optic neuropathy with obvious visual field defects, and is the most common irreversible blinding eye disease in the world $(1,2)$. By the end of 2020, about 79.6 million people will have glaucoma (3). Primary angle-closure glaucoma (PACG) is the most common type of glaucoma in China; however, the exact pathogenesis is still unclear. Currently, identified risk factors include elevated intraocular pressure (IOP) (4), metabolic abnormalities (5), and family history of glaucoma (6). However, other potential risk factors for PACG may also exist; therefore, exploring these potential factors to develop interventions to reduce the incidence of glaucoma is of the utmost urgency.

Recent epidemiological studies have suggested that hyperlipidemia may be associated with glaucoma, but the findings are contradictory. Newman-Casey et al. (7) found that people with hyperlipidemia had a lower risk of primary open angle glaucoma (POAG) than those without hyperlipidemia. However, one study by Lin et al. (8) showed that there was a positive correlation between hyperlipidemia and the development of glaucoma. Hyperlipidemia would increase the risk of glaucoma, and elevated lipid levels were also considered to be a risk factor for increased IOP. The results of Yilmaz et al. (9) also suggested that the pathogenesis of different types of glaucoma may be related to changes in hyperlipidemia.

At present, there are limited relevant studies to explore the association between lipid levels and PACG. Therefore, we performed a cross-sectional, case-control study to clarify the association between lipid levels and PACG.

\section{MATERIALS AND METHODS}

\section{Subjects}

The study was approved by the Ethics Committee of the Eye and ENT Hospital of Fudan University, Shanghai, China. It was conducted in accordance with the Declaration of Helsinki. Informed consent was obtained from all subjects. Patients with PACG were consecutively recruited from the Department of Ophthalmology and Visual Science, Eye and ENT Hospital of Fudan University between November 2018 and August 2020.

Control subjects were consecutively recruited from individuals who participated in annual health screenings during the study period. The study clerks conducted face-toface questionnaire interviews to gather data, such as diabetes, hypertension, height, and body weight.

We used an open source calculator to calculate the minimal required sample size based on the probability of a type I error of alpha at 5\% (two-sided), type II error of beta at $20 \%$, the Odds Ratio at 2.5, a sample size ratio of 0.8 , the normal population have prevalence of PACG that is about $5 \%$ in China. This calculation yielded a sample consisting of at least 272 cases and 218 controls.

\section{Examination}

Medical examinations for all subjects included an electrocardiogram, X-ray, and an assessment of liver function, kidney function, infectious disease, blood pressure, heart rate, and body temperature. All subjects underwent medical examinations at the Eye and ENT Hospital of Fudan University. Each PACG patient also underwent a standardized ophthalmic examination performed by glaucoma specialists. The methods used to perform the ophthalmic examinations have been previously described in detail (10-13).

Laboratory testing was performed at the Department of Clinical Laboratory, Eye and ENT Hospital of Fudan University. Blood samples were obtained in the morning after subjects fasted for $8 \mathrm{~h}$. The tubes were centrifuged for $10 \mathrm{~min}$ at $3,000 \mathrm{r} / \mathrm{min}$. Serum high-density lipoprotein cholesterol (HDL-C), low-density lipoprotein cholesterol (LDLC), small dense LDL-C (SDLDL-C), triglyceride (TRIG), and cholesterol (CHOL) levels were measured by enzymatic colorimetry (Roche Cobas 8000C702, Mannheim, Germany). Serum apolipoprotein A (APOA), apolipoprotein B (APOB), apolipoprotein E (APOE), and lipoprotein(a) (LPa) levels were measured by immunoturbidimetry (Roche Cobas 8000C702, Mannheim, Germany). In order to ensure the accuracy of the detection system, the biochemical analyzer conduct an indoor quality control test every day, and the monthly coefficient of variation was controlled within the range of $3-5 \%$.

\section{Diagnostic and Inclusion Criteria PACG Subjects}

PACG was diagnosed by a glaucoma specialist. Previously described diagnostic criteria were used to diagnose PACG and previously described inclusion criteria were used to select patients (11-13). PACG was diagnosed based on narrow anterior chamber angles with glaucomatous optic neuropathy and corresponding VF loss. VF loss was determined by including a cluster of three or more non-edge contiguous points on the pattern deviation plot, which did not cross the horizontal meridian, and had a probability of $<5 \%$ of being present in agematched controls (one of which was $<1 \%$ ); it had an abnormal standard deviation pattern with a $p<0.05$ occurring in the normal population, and fulfilled the following test reliability criteria: $<20 \%$ fixation losses, $<15 \%$ false positives, and/or $<15 \%$ false negatives. Additionally, PACG was diagnosed in eyes with narrow angles, elevated IOP (IOP $>21 \mathrm{mmHg}$ ), having at least $180^{\circ}$ of angle-closure obliterating the pigmented segment of the trabecular meshwork, whether synechial, appositional, segmented, or continuous, and in cases where the degree of peripheral anterior synechiae was too extensive to be managed by laser peripheral iridotomy. The inclusion criteria for PACG subjects were as follows: absence of any secondary glaucoma or any other eye disease that could potentially affect visual acuity or the VF, absence of any intraocular surgery within the previous 2 months, absence of any systemic diseases including acute infectious diseases, metabolic syndrome, autoimmune disease, or cancer.

A total of 414 participants were recruited, of whom 94 were later excluded (secondary glaucoma $=60$, congenital glaucoma $=12$, neovascular glaucoma $=10$, cataract $=5$, systemic diseases $=5$, macular degeneration $=2$ ), leaving a final sample of 320 patients (newly diagnosed PACG patients $=202$, referral PACG patients = 118). "Newly diagnosed PACG patients" mean that 
they reported themselves that they have no treatment experience before coming to our hospital. They were first diagnosed with glaucoma at our hospital.

\section{Control Subjects}

The normal subjects were recruited from health screenings, and without eye discomfort. Moreover, each normal control subject underwent preliminary ophthalmic examinations, which included refractive status, gonioscopy, and slit-lamp biomicroscopic examination, performed by glaucoma specialists. The inclusion criteria for normal subjects were as follows: absence of any type of glaucoma or any other eye disease that could potentially affect visual acuity or the VF, absence of any intraocular surgery within the previous 2 months, absence of any systemic diseases including acute infectious diseases, metabolic syndrome, autoimmune disease, or cancer. A total of 277 control subjects were recruited, of whom 35 subjects were later excluded from the study based on the inclusion criteria (cataract $=8$, cancer $=2$, renal disease $=6$, liver disease $=4$, autoimmune disease $=2$, macular degeneration $=3$, cardiovascular disease $=4$, other diseases $=6$ ), leaving a final sample of 242 control subjects. There were no significant difference of demographics and disease severity $(p>0.05)$ between newly diagnosed PACG patients and referral PACG patients (Supplementary Table 1).

\section{Statistical Analyses}

The analyses were performed using version 13.0 of the Statistical Package for the Social Sciences (SPSS Inc., Chicago, IL, USA). The results are presented as mean \pm standard deviation (SD). Normality was assessed using the KolmogorovSmirnoff test. The independent Student $t$-test and chi-squared test were used to compare the subject characteristics between the groups. One-way analysis of variance (ANOVA) was used to compare the lipid levels among the three groups. Correlations between lipid levels and ocular parameters were assessed using Pearson correlation analysis. Multivariate linear regression analyses were performed to evaluate the association between lipid levels and ocular parameters. Furthermore, logistic regression analyses were performed to identify the risk factors for PACG. A two-sided $p$-value of $<0.05$ was considered statistically significant.

\section{RESULTS}

\section{Characteristics of the Study Patients}

A total of 320 subjects with PACG (male $=146$, female $=174)$ and 242 control subjects (male $=111$, female $=131$ ) were recruited in this study. There was no statistical difference in the mean age and sex between the PACG and control subjects $(P>$ 0.05). The serum LDL-C, TRIG, HDL-C, APOE, LPa, CHOL, APOB, and APOA levels were significantly higher $(p<0.05)$ in the PACG group than in the control group. Table 1 presents a summary of the demographics and serum lipid levels of the PACG and control groups.

\section{Comparison of Serum Lipid Levels in PACG Subjects in the Sex- and Age-based Subgroups}

The PACG subjects were categorized into sex-based female and male subgroups. In both the female and male subgroups, the subjects were further divided into two groups based on age ( $>60$ subgroup, $\leq 60$ subgroup). In the male subgroup $\leq 60$ years, the mean serum TRIG, APOE, and LPa levels were significantly higher $(P<0.005)$ in the PACG group compared to the control group. In the male subgroup $>60$ years, the mean serum HDL$\mathrm{C}$ and APOE levels were significantly higher in the PACG group compared to the control group (Supplementary Table 2).

In the female subgroup $\leq 60$ years, the mean serum HDLC, SDLDL-C, APOA, APOB, APOE, and CHOL levels were significantly higher $(P<0.005)$ in the $P A C G$ group compared to the control group. In the female subgroup $>60$ years, the mean serum LDL-C, SDLDL-C, APOA, APOB, APOE, LPa, and CHOL levels were significantly higher $(P<0.005)$ in the PACG group compared to the control group (Supplementary Table 3).

\section{Association Between Serum Lipids and Ocular Parameters}

Pearson correlation analysis was carried out between the serum lipid levels and ocular parameters. Pearson analysis showed that the serum CHOL, HDL-C, LDL-C, APOA, and APOB levels were positively correlated with the mean deviation (MD) level $(r=$ $0.247, P<0.001 ; r=0.202, P<0.001 ; r=0.229, P<0.001 ; r$ $=0.218, P<0.001 ; r=0.175, P<0.05$, respectively; Table 2).

\section{Logistic Regression Analysis of the Association Between Serum Lipid Levels in the PACG and Control Subjects}

Logistic regression analyses revealed that serum HDL-C (odds ratio $[\mathrm{OR}]=11.01,95 \%$ confidence interval $[\mathrm{CI}]=5.616-$ 21.587), SDLDL-C (OR $=1.007,95 \% \mathrm{CI}=1.005-1.008), \mathrm{APOA}$ $(\mathrm{OR}=13.621,95 \% \mathrm{CI}=7.251-25.591), \mathrm{APOB}(\mathrm{OR}=2.243$, $95 \% \mathrm{CI}=1.060-4.732), \mathrm{LPa}(\mathrm{OR}=0.999,95 \% \mathrm{CI}=0.998-$ 1.00 ), and $\mathrm{CHOL}$ levels ( $\mathrm{OR}=1.131,95 \% \mathrm{CI}=1.005-1.326)$ were associated with PACG after adjusting for age and other demographic parameters (Table 3).

\section{Multiple Linear Regressions for Associations Between Lipid Levels and MD in PACG Subjects}

Multiple linear regression analysis, adjusting for age, sex, body mass index, systolic blood pressure, diastolic blood pressure, hypertension, diabetes, IOP, central corneal thickness, anterior chamber depth, and axial length was performed to further analyze the association between serum lipid levels and MD (Table 4). There was a statistically correlation between HDLC levels and $\mathrm{MD}(B=0.389, P=0.002,95 \% \mathrm{CI}=-1.249$ to $-0.624)$; LDL-C levels and MD $(B=0.190, P=0.019,95 \% \mathrm{CI}$ $=-5.632$ to -1.306$)$; and CHOL levels and $\mathrm{MD}(B=0.364, P=$ $0.027,95 \% \mathrm{CI}=-7.722$ to -1.839 ). 
TABLE 1 | Demographics parameters and serum lipids levels of the PACG and control groups.

\begin{tabular}{|c|c|c|c|c|}
\hline & PACG group & Control group & $T$ value & $P$-value \\
\hline No. & 320 & 242 & & \\
\hline Age (years) & $60.5 \pm 11.8$ & $58.9 \pm 8.88$ & 1.755 & 0.08 \\
\hline Gender (Male/Female) & $146 / 174$ & $111 / 131$ & 0.057 & 0.95 \\
\hline Diabetes (Yes/No) & $27 / 293$ & $15 / 227$ & 0.999 & 0.318 \\
\hline Hypertension (Yes/No) & $96 / 224$ & $61 / 181$ & 1.573 & 0.210 \\
\hline $\mathrm{BMI}\left(\mathrm{Kg} / \mathrm{m}^{2}\right)$ & $22.73 \pm 3.4$ & $23.47 \pm 5.78$ & 1.019 & 0.309 \\
\hline IOP (mm Hg) & $21.46 \pm 11.16$ & - & & \\
\hline VCDR & $0.63 \pm 0.28$ & - & & \\
\hline $\mathrm{ACD}(\mathrm{cm})$ & $1.93 \pm 0.48$ & - & & \\
\hline CCT (mm) & $544.30 \pm 40.86$ & - & & \\
\hline LDL-C (mmol/L) & $2.67 \pm 0.79$ & $2.51 \pm 0.58$ & 2.813 & 0.005 \\
\hline TRIG (mmol/L) & $1.79 \pm 1.31$ & $1.51 \pm 0.97$ & 2.859 & 0.004 \\
\hline HDL-C (mmol/L) & $1.21 \pm 0.34$ & $0.96 \pm 0.21$ & 9.764 & $<0.001$ \\
\hline SDLDL-C (mmol/L) & $0.34 \pm 0.29$ & $0.35 \pm 0.18$ & 0.325 & 0.745 \\
\hline APOA (mmol/L) & $1.49 \pm 0.31$ & $1.23 \pm 0.27$ & 10.32 & $<0.001$ \\
\hline APOB (mmol/L) & $0.92 \pm 0.23$ & $0.81 \pm 0.19$ & 6.358 & $<0.001$ \\
\hline APOE (mmol/L) & $39.27 \pm 10.5$ & $34.6 \pm 9.52$ & 5.42 & $<0.001$ \\
\hline $\mathrm{LPa}(\mathrm{mmol} / \mathrm{L})$ & $189.28 \pm 20.1$ & $138.7 \pm 16.1$ & 3.125 & 0.002 \\
\hline $\mathrm{CHOL}(\mathrm{mmol} / \mathrm{L})$ & $4.71 \pm 1.01$ & $4.10 \pm 0.77$ & 7.682 & $<0.001$ \\
\hline
\end{tabular}

$V C D R$, vertical cup/disc ratio; CCT, central corneal thickness; $A C D$, anterior chamber depth; AL, axial length; MD, mean deviation values for the visual field; MS, mean sensitivity values for the visual field; IOP, intraocular pressure; BMI, body mass index; SBP, systolic blood pressure; DBP, diastolic blood pressure; PACG, primary angle closure glaucoma; HDL-C, high-density lipoprotein cholesterol; LDL-C, low-density lipoprotein cholesterol; SDLDL-C, small dense low-density lipoprotein cholesterol; TRIG, triglyceride; CHOL, cholesterol; APOA, apolipoprotein A; APOB, apolipoprotein B; APOE, apolipoprotein E; Lpa, lipoprotein a.

TABLE 2 | Association between serum lipids and ocular parameters.

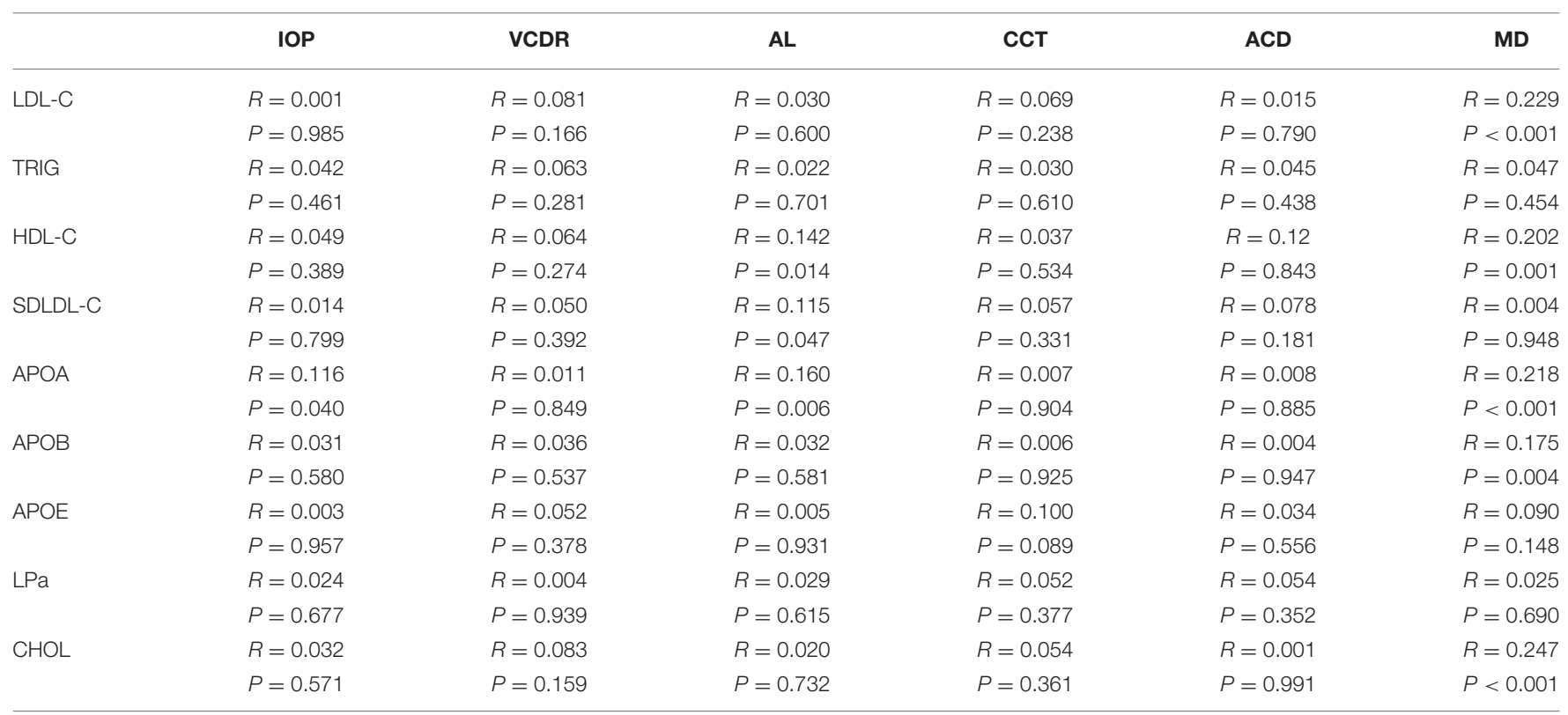

VCDR, vertical cup/disc ratio; CCT, central corneal thickness; $A C D$, anterior chamber depth; AL, axial length; MD, mean deviation values for the visual field; IOP, intraocular pressure; HDL-C, high-density lipoprotein cholesterol; LDL-C, low-density lipoprotein cholesterol; SDLDL-C, small dense low-density lipoprotein cholesterol; TRIG, triglyceride; CHOL, cholesterol; $A P O A$, apolipoprotein A; $A P O B$, apolipoprotein B; $A P O E$, apolipoprotein E; Lpa, lipoprotein a. 
TABLE 3 | Logistic regression analysis of the association between serum lipid levels and PACG.

\begin{tabular}{|c|c|c|c|c|}
\hline & $B$ & $P$ & OR & $95 \% \mathrm{Cl}$ \\
\hline LDL-C & 0.285 & 0.008 & 1.330 & $1.079-1.640$ \\
\hline TRIG & 0.025 & 0.501 & 1.025 & $0.953-1.103$ \\
\hline HDL-C & 2.399 & $<0.001$ & 11.01 & $5.616-21.587$ \\
\hline SDLDL-C & 0.007 & $<0.001$ & 1.007 & $1.005-1.008$ \\
\hline APOA & 2.612 & $<0.001$ & 13.621 & $7.251-25.591$ \\
\hline APOB & 0.808 & 0.034 & 2.243 & $1.060-4.732$ \\
\hline APOE & 0.013 & 0.094 & 1.104 & 0.998-1.030 \\
\hline $\mathrm{LPa}$ & 0.001 & 0.030 & 0.999 & $0.998-1.00$ \\
\hline $\mathrm{CHOL}$ & 0.123 & $<0.001$ & 1.131 & $1.005-1.326$ \\
\hline
\end{tabular}

Adjusted for age, gender, BMI, diabetes, hypertension, systolic blood pressure (SBP), diastolic blood pressure (DBP). HDL-C, high-density lipoprotein cholesterol; LDL-C, lowdensity lipoprotein cholesterol; SDLDL-C, small dense low-density lipoprotein cholesterol; TRIG, triglyceride; $C H O L$, cholesterol; $A P O A$, apolipoprotein A; APOB, apolipoprotein B; APOE, apolipoprotein E; Lpa, lipoprotein a.

TABLE 4 | Multiple linear regressions for associations between lipid levels in patients with PACG.

\begin{tabular}{lccc}
\hline & $\boldsymbol{B}$ & $\boldsymbol{P}$ & $\mathbf{9 5 \%} \mathbf{C l}$ \\
\hline LDL-C & 0.190 & 0.019 & -5.632 to -1.306 \\
TRIG & 0.052 & 0.512 & -8.175 to 4.139 \\
HDL-C & 0.389 & 0.002 & -1.249 to -0.624 \\
SDLDL-C & 0.011 & 0.886 & -15.303 to -3.517 \\
APOA & 0.089 & 0.555 & -10.313 to 5.552 \\
APOB & 0.013 & 0.931 & -10.980 to 10.050 \\
APOE & 0.034 & 0.598 & -0.126 to 0.073 \\
LPa & 0.057 & 0.346 & -0.007 to 0.002 \\
CHOL & 0.364 & 0.027 & -7.722 to -1.839 \\
\hline
\end{tabular}

Adjusted for age, gender, BMI, diabetes, hypertension, systolic blood pressure, diastolic blood pressure. HDL-C, high-density lipoprotein cholesterol; LDL-C, low-density lipoprotein cholesterol; SDLDL-C, small dense low-density lipoprotein cholesterol; TRIG, triglyceride; $C H O L$, cholesterol; APOA, apolipoprotein A; APOB, apolipoprotein B; APOE, apolipoprotein E; Lpa, lipoprotein a.

\section{DISCUSSION}

The following points were found in our research. First, the serum LDL-C, TRIG, HDL-C, APOE, LPa, CHO, APOB, and APOA levels were higher in the PACG patients. Second, the serum CHOL, HDL-C, LDL-C, APOA, and APOB levels were positively correlated with the MD level. Finally, high serum HDL-C, SDLDL-C, APOA, APOB, Lpa, and CHOL levels were associated with PACG. The results of our study indicate that higher serum HDL-C, SDLDL-C, APOA, APOB, and LPa levels are associated with a significantly increased risk of PACG. These findings are consistent with the literature $(7,14)$.

The pathogenesis of PACG is complex; shallow anterior chamber, short axial length, hyperopia, and east Asian ethnic origin are the main risk factors for primary angle-closure glaucoma $(6,15)$. Furthermore, abnormal lipid metabolism is an important risk factor for cardiovascular disease and is associated with many eye diseases (16). In addition, a large number of studies have shown that abnormal lipid metabolism hyperlipidemia is associated with glaucoma (8). The study of Edwards et al. (17) indicated that endogenous lipids may be involved in the regulation of IOP homeostasis. Furthermore, Tang et al. (18) showed that TRIG were higher, and was an independent risk factor for POAG. Other studies have also confirmed the association between LDL-C and glaucoma $(9,19)$. Yilmaz et al. (9) also found increased TRIG and CHOL levels in normal intraocular pressure glaucoma. Lee et al. (20) found that hyperlipidemia had a certain correlation with the occurrence and development of POAG. One study (21) found that glaucomalike pathological changes occurred in the eyes of rabbits fed a high-fat diet. Other studies (22-24) also found that the lipid levels and types of aqueous humor and trabecular mesh in glaucoma animal models and patients were significantly different from those in the control group, indicating that lipid levels were correlated with glaucoma. Our results also indicate that hyperlipidemia has a certain correlation with the occurrence and severity of PACG.

Currently, the mechanism by which hyperlipidemia increases the risk of glaucoma onset and progression is unclear. Ishikawa et al. (25) showed that high serum lipid levels would increase peripheral venous pressure and blood viscosity, leading to a decrease in blood outflow. Wang et al. (16) found that lipid metabolic abnormalities can change hemodynamics and affect the aqueous humor outflow, which can lead to elevated IOP. Other scholars have also confirmed that hyperlipidemia can increase blood viscosity, in turn, affecting the aqueous humor circulation pathway, leading to an elevated intraocular pressure, which may lead to glaucoma $(26,27)$. Moreover, increased serum lipid levels might cause degenerative changes in ocular blood vessels, leading to increased production of reactive oxygen species, and thus accelerate the development of glaucoma (24). Kim et al. (28) found that increased LDL-C levels can increase blood viscosity, thereby affecting the microcirculation of the eye, and thus participate in the development of glaucoma.

In this study, the level of LDL-C was higher in older female PACG patients, but not in younger female PACG patients. The reason for this finding may be explained by menopausal status differences. Schaefer et al. (29) reported that increased age was associated with higher plasma LDL-C levels, especially in women. After adjustment for age and BMI, LDL-C level was still significantly higher in older female PACG patients than in younger female PACG patients, indicating a hormonal effect on LDL metabolism.

Moreover, the level of HDL-C was significantly higher in younger female PACG patients, but not in older female PACG patients. From our results, the following two questions arise: first, why is the serum concentration of HDL-C higher in subjects with PACG? Traditionally, HDL-C is considered as protective for cardiovascular disease and also known as "good cholesterol"; Second, what could be the reason behind the difference in the serum levels of HDL-C between younger and older female subjects with PACG when compared with the control groups? Limited data are available in the literature regarding these results. The reasons for these findings are currently not well-understood. 
Further research is needed to better understand the complex relationship and possible mechanism between serum lipid level and PACG.

Furthermore, significantly higher levels of fewer types of lipids in males compared to female subjects were observed in this study. The explanation for this may be explained by sex differences in obesity, and physical activity (30-32). For example, Saquib et al. (30) reported that the prevalence of both generalized (79 vs. $53 \%$ ) and central obesity ( 85 vs. $42 \%$ ) were significantly higher in women than men. Women reported spending more time watching TV and spending less time walking than men $(p<$ $0.05)$. Qiao et al. (31) reported that women were more likely than men to have diabetes and obesity, and to have higher CHOL, and LDL-C levels.

Our research has certain limitations. First, our study is a crosssectional, case-control study, and the ability to explore the exact mechanism of the association between serum lipid levels and PACG is still insufficient. This, in turn, limits our ability to make accurate inferences about the causal relationship between serum lipid levels and PACG. Second, the subjects involved in this study were Chinese, which may limit the universality of the results. Finally, we did not consider the treatment of lipid-lowering drugs and how they might affect the test results. Therefore, in the future, multi-center, prospective research with large sample sizes should be carried out.

In conclusion, the results of this study showed that the serum LDL-C, TRIG, HDL-C, APOE, LPa, CHOL, APOB, and APOA levels in PACG patients were significantly higher than those in the control group. Higher serum HDL-C, SDLDL-C, APOA, APOB, and LPa levels were significantly correlated with PACG. Therefore, the exploration of treatment methods from the lipid level will open up a new direction for the treatment of PACG. However, a multi-center, in-depth investigation of clinical studies is needed for further research and demonstration.

\section{REFERENCES}

1. Bourne RR, Stevens GA, White RA, Flaxman SR, Price H, Jonas JB, et al. Causes of vision loss worldwide, 1990-2010: a systematic analysis. Lancet Glob Health. (2013) 1:e339-49. doi: 10.1016/S2214-109X(13) 70113-X

2. Tham YC, Li X, Wong TY, Quigley HA, Aung T, Cheng CY. Global prevalence of glaucoma and projections of glaucoma burden through 2040: a systematic review and meta-analysis. Ophthalmology. (2014) 121:208190. doi: 10.1016/j.ophtha.2014.05.013

3. Quigley HA, Broman AT. The number of people with glaucoma worldwide in 2010 and 2020. Br J Ophthalmol. (2006) 90:2627. doi: 10.1136/bjo.2005.081224

4. Baskaran M, Foo RC, Cheng CY, Narayanaswamy AK, Zheng YF, Wu $\mathrm{R}$, et al. The prevalence and types of glaucoma in an urban Chinese population: the Singapore Chinese Eye Study. JAMA Ophthalmol. (2015) 133:874-80. doi: 10.1001/jamaophthalmol.2015.1110

5. Chen HY, Lin CL. Comparison of medical comorbidity between patients with primary angle-closure glaucoma and a control cohort: a population-based study from Taiwan. BMJ Open. (2019) 9:e024209. doi: 10.1136/bmjopen-2018-024209

6. Jonas JB, Aung T, Bourne RR, Bron AM, Ritch R, Panda-Jonas S. Glaucoma. Lancet. (2017) 390:2183-93. doi: 10.1016/S0140-6736(17)31469-1

\section{DATA AVAILABILITY STATEMENT}

The raw data supporting the conclusions of this article will be made available by the authors, without undue reservation.

\section{ETHICS STATEMENT}

The studies involving human participants were reviewed and approved by the Ethics Committee of the Eye and ENT Hospital of Fudan University. The patients/participants provided their written informed consent to participate in this study. Written informed consent was obtained from the individual(s) for the publication of any potentially identifiable images or data included in this article.

\section{AUTHOR CONTRIBUTIONS}

SL, MS, and WC conceived the study and participated in drafting the final manuscript. SL, YL, JT, MS, and WC analyzed the data and completed the final draft of the manuscript. All authors have read and approved the manuscript.

\section{FUNDING}

This work was supported by Shanghai Sailing Program (18YF1403500), Shanghai Municipal Commission of Health and Family Planning (201840050), and Shanghai Science and Technology Committee Foundation Grant (19411964600).

\section{SUPPLEMENTARY MATERIAL}

The Supplementary Material for this article can be found online at: https://www.frontiersin.org/articles/10.3389/fmed. 2021.618970/full\#supplementary-material

7. Newman-Casey PA, Talwar N, Nan B, Musch DC, Stein JD. The relationship between components of metabolic syndrome and open-angle glaucoma. Ophthalmology. (2011) 118:1318-26. doi: 10.1016/j.ophtha.2010.11.022

8. Lin HC, Chien CW, Hu CC, Ho JD. Comparison of comorbid conditions between open-angle glaucoma patients and a control cohort: a case-control study. Ophthalmology. (2010) 117:2088-95. doi: 10.1016/j.ophtha.2010.03.003

9. Yilmaz N, Coban DT, Bayindir A, Erol MK, Ellidag HY, Giray O, et al. Higher serum lipids and oxidative stress in patients with normal tension glaucoma, but not pseudoexfoliative glaucoma. Bosn J Basic Med Sci. (2016) 16:21-7. doi: 10.17305/bjbms.2016.830

10. Li S, Chen Y, Shao M, Tang L, Sun X, Cao W. Association of plasma complement C3 levels with primary angle-closure glaucoma in older women. Invest Ophthalmol Vis Sci. (2017) 58:682-9. doi: 10.1167/iovs.16-20675

11. Li S, Shao M, Cao W, and Sun X. Association between pretreatment serum uric acid levels and progression of newly diagnosed primary angle-closure glaucoma: a prospective cohort study. Oxidat Med Cell Longev. (2019) 2019:7919836. doi: 10.1155/2019/7919836

12. Li S, Shao M, Wan Y, Tang B, Sun X, Cao W. Relationship between ocular biometry and severity of primary angle-closure glaucoma: relevance for predictive, preventive, and personalized medicine. EPMA J. (2019) 10:26171. doi: 10.1007/s13167-019-00174-1

13. Li S, Zhang H, Shao M, Li Y, Song Y, Sun X, et al. Association between 17$\beta$-estradiol and interleukin-8 and visual field progression in postmenopausal 
women with primary angle closure glaucoma. Am J Ophthalmol. (2020) 217:55-67. doi: 10.1016/j.ajo.2020.04.033

14. Kim M, Jeoung JW, Park KH, Oh WH, Choi HJ, Kim DM. Metabolic syndrome as a risk factor in normal-tension glaucoma. Acta Ophthalmol. (2014) 92:e637-43. doi: 10.1111/aos.12434

15. Xinghuai S, Yi D, Yuhong C, Dao-Yi Y, Stephen JC, Junyi C, et al. Primary angle closure glaucoma: what we know and what we don't know. Prog Retin Eye Res. (2017) 57:26-45. doi: 10.1016/j.preteyeres.2016.12.003

16. Wang S, Xu L, Jonas JB, You QS, Wang YX, Yang H, et al. Dyslipidemia and eye disease in the adult Chinese population: the Beijing Eye Study. PLoS ONE. (2012) 7:e26871. doi: 10.1371/journal.pone.0026871

17. Edwards G, Arcuri J, Wang H, Ziebarth N, Zode G, Lee RK, et al. Endogenous ocular lipids as potential modulators of intraocular pressure. J Cell Mol Med. (2020) 24:3856-900. doi: 10.1111/jcmm.14975

18. Tang B, Shao M, Li S. Association between blood lipid level and primary open angle glaucoma. Chin J Lab Med. (2017) 40:206-11. doi: 10.3760/cma.j.issn.1009-9158.2017.03.014

19. Modrzejewska M, Grzesiak W, Zaborski D, Modrzejewska A. The role of lipid dysregulation and vascular risk factors in Glaucomatous retrobulbar circulation. Bosn J Basic Med Sci. (2015) 15:50-6. doi: 10.17305/bjbms.2015.299

20. Lee SH, Kim GA, Lee W, Bae HW, Seong GJ, Kim CY. Vascular and metabolic comorbidities in open-angle glaucoma with low- and high-teen intraocular pressure: a cross-sectional study from South Korea. Acta Ophthalmol. (2017) 95:e564-74. doi: 10.1111/aos.13487

21. Kashiwagi E, Masuno K, Fujisawa K, Matsushima S, Torii M, Takasu N. Glaucoma in a New Zealand white rabbit fed high-cholesterol diet. J Toxicol Pathol. (2012) 25:51-3. doi: 10.1293/tox.25.51

22. Mayordom-Febrer A, Lopez-Murciam, Morales-Tatay JM, Monleon-Salvado D, Pinazo-Duran MD. Metabolomics of the aqueous humor in the rat glaucoma model induced by a series of intracamerular sodium hyaluronate injection. Exp Eye Res. (2015) 131:84-92. doi: 10.1016/j.exer.2014.11.012

23. Aribindi K, Guerray, Lee RK, Bhattacharya SK. Comparative phospholipid profiles of control and glaucomatous human trabecular meshwork. Invest Ophthalmol Vis Sci. (2013) 54:3037-44. doi: 10.1167/iovs.12-10517

24. Aribindi K, Guerra Y, Piqueras MC, Banta JT, Lee RK, Bhattacharya SK. Cholesterol and glycosphingo lipids of human trabecular and glaucomatous donors. Curr Eye Res. (2013) 38:1017-26. doi: 10.3109/02713683.2013.803123

25. Ishikawa M, Sawada Y, Sato N, Yoshitomi T. Risk factors for primary openangle glaucoma in Japanese subjects attending community health screenings. Clin Ophthalmol. (2011) 5:1531-7. doi: 10.2147/OPTH.S26049
26. Tan GS, Wong TY, Fong CW, Singapore Malay Eye Study. Diabetes, metabolic abnormalities, and glaucoma. Arch Ophthalmol. (2009) 127:135461. doi: 10.1001/archophthalmol.2009.268

27. Kim YH, Jung SW, Nam GE, Han KD, Bok AR, Baek SJ, et al. High intraocular pressure is associated with cardiometabolic risk factors in South Korean men: Korean National Health and Nutrition Examination Survey, 2008-2010. Eye (Lond). (2014) 28:672-9. doi: 10.1038/eye.2014.43

28. Kim MJ, Kim HS, Kim HS, Jeoung JW, Park KH. Risk factors for open-single glaucoma with normal baseline intraocular pressure in a young population: the Korea Nation Health and nutrition Examination Survey. Clin Exp Ophthalmol. (2014) 42:825-32. doi: 10.1111/ceo. 12347

29. Schaefer EJ, Lamon-Fava S, Cohn SD, Schaefer MM, Ordavas JM, Castelli WP, et al. Effects of age, gender, and menopausal status on plasma low density lipoprotein cholesterol and apolipoprotein B levels in the Framingham Offspring Study. J Lipid Res. (1994) 35:77992. doi: 10.1016/S0022-2275(20)39173-2

30. Saquib J, Saquib N, Stefanick ML, Khanam MA, Anand S, Rahman M, et al. Sex differences in obesity, dietary habits, and physical activity among urban middle-class Bangladeshis. Int J Health Sci (Qassim). (2016) 10:36372. doi: $10.12816 / 0048730$

31. Qiao Q, Hong Y, Zhao WJ, Zhou G, Liu Q, Ning X, et al. Sex differences in outcomes and associated factors among stroke patients with small artery occlusion in China. Biol Sex Differ. (2018) 9:35. doi: 10.1186/s13293-018-0194-6

32. Sorkin DH, Biegler KA, Billimek J. Differences in self-reported physical activity and body mass index among older Hispanic and non-Hispanic white men and women: findings from the 2009 California Health Interview Survey. J Am Geriatr Soc. (2015) H 63:2158-63. doi: 10.1111/jgs.13655

Conflict of Interest: The authors declare that the research was conducted in the absence of any commercial or financial relationships that could be construed as a potential conflict of interest.

Copyright (C) 2021 Shao, Li, Teng, Li and Cao. This is an open-access article distributed under the terms of the Creative Commons Attribution License (CC BY). The use, distribution or reproduction in other forums is permitted, provided the original author(s) and the copyright owner(s) are credited and that the original publication in this journal is cited, in accordance with accepted academic practice. No use, distribution or reproduction is permitted which does not comply with these terms. 